\title{
The Architectural Instrumentalist - exploring spatio-temporal interdependence in the composition of performed music and architectural space.
}

\author{
Emma-Kate Matthews \\ University College London
}

\begin{abstract}
:
Sound, space and time are inextricably linked. As an architect and musician, I am interested in the simultaneous capacities of architecture to influence the composition and experience of performed music - and of performed music to influence the composition and experience of architectural space. My architectural research project the Augmented Instrumentalist (tAl) - sets out a methodology by which architectural spaces are understood as and designed to be instruments - as a means of replicating the unique acoustic profile of the city of Venice during the acqua alta. In tandem with the development of acoustically generative themes within my architectural research; my musical compositions actively appropriate architectural spaces as musical instruments, by responding to the acoustic qualities of specific performance spaces. When performed, my pieces exploit acoustical phenomena such as reverberance / echo and sympathetic resonance to articulate and augment musical motifs. For example, by using excessive reverberance to construct a harmonic sequence, or by suggesting a rhythm from an echo. Architectural acoustics have long had an effect on the development of musical ideas: "... in the old churches the walls were in fact powerful instruments which the ancients learned to play upon... [When it was discovered that] more than one tone could be heard at the same time with pleasing results, the harmonies produced by the coinciding of notes began to be regulated and used. From this partsinging developed [polyphonic music]" (Rasmussen, 1962: 230)
\end{abstract}

This article investigates spatio-temporal interdependence between architecture and performed music within the context of my ongoing research.

\section{Key Words:}

Spatial mediators

Spatialised score

Music performance

Acoustic correspondence

Spatio-temporal interdependence

Resonance

Performance

Site-Specific Composition

Instrumental Architecture

\section{Nostalgic resonance:}

My fascination with acoustics became intensified during a trip to Venice. The city of Venice has a unique acoustic character. On the occasions when the evening bells and the acqua alta ${ }^{1}$ coincide - the seemingly infinite reflections of light and sound envelop the city and induce a hypnagogic-like state in anyone lucky enough to be

\footnotetext{
${ }^{1}$ Acqua alta translates from Italian as "high water" - and occurs very regularly in Venice, when certain meteorological conditions coincide. the phenomenon is most likely to occur during the winter months. An eerie siren sounds throughout the city to warn its residents in advance of the flood.
} 
present. Such intense immersive qualities are a direct consequence of both the geometry and the materiality of the city. The tightly packed calli² are lined with acoustically reflective materials, including the carpet of seawater which lays claim to the ground floor during the acqua alta.

“Reflectance Profile 001: Chioggia”

Acoustic reflections linger and merge with each other as they bounce between the walls of the narrow streets, and it becomes near-impossible to spatially locate the bells which generate and dominate the Venetian soundscape. Venice is simultaneously an instrument, a performer and the stage on which these two unite, to perform. Venice is a city which challenges and redefines conventionally understood boundaries between these elements. Similarly, my work operates within the thresholds between these performative agents, with a view to de-opposing the practices of architectural and musical composition, and to define a reciprocality between the composition and execution of music and architectural space.

I refer to this correspondence - in my own work - as 'resonance'. This term implies relationships between multiple parts in which compatibilities are identified and each part is charged with the ability to influence and interact with the others. This system is distinct from one where each part maintains a distinct level of autonomy by attempting to convert one into the other, by means of mathematical or metaphorical analogy. Many architects and musicians experience desires to form connections between their respective worlds - often by looking for similarities between ideas, or by abstracting ideas held in one and then translating those abstractions to describe the ideas of the other:

Johann Wolfgang Von Goethe once reportedly stated that "Architecture [is] frozen music... the influence that flows upon us from architecture is like that from music" (Goethe, 1839: 282) - Goethe's metaphorical translation from an experience of music into an architectural gesture is certainly evocative, but arguably also frustratingly abstract. His description suggests that if the time-based medium of music was to become frozen in time, its very essence would transform into the spatial, static medium of architecture: However, the way we navigate spaces and hear music is inescapably linear and temporal with regard to experience and perception. Goethe's quote becomes more interesting when we consider his use of the word "influence" - though in the context of composition rather than experience. Countless renaissance architects and musicians have shared ideologies of proportion and scale in their definitions of geometric or harmonic and rhythmic systems in the composition of space and music respectively. Musicologist Charles Warren identified this reciprocity with respect to the work of 15th century Italian designer Brunelleschi. Warren states that Brunelleschi was one of the first renaissance architects to interrogate mathematical principles underlying musical consonances and reapply these principles as drivers for the perspectival organisation of spatial relationships. (Warren, 1973: 93) Warren also proposes that the numeric relationships which defined Brunelleschi's geometric set-out of the great cupola of the Cattedrale di Santa Maria del Fiore were used to determine mensural proportions in the composition of "Nuper Rosarum Flores" - a motet written by chorister Guillarme Dufay to mark the opening of the Cathedral (Evans, 2000: 243). It is now interesting to test the sentiment of Goethe's statement in reverse: That architecture (or frozen music) becomes temporally liberated when the numeric principles governing its geometric fundamentals are appropriated in the definition and development of harmonic, melodic and rhythmic themes. At this point, it's also interesting to note Goethe's use of the word "flow" - in parallel to acknowledging

\footnotetext{
${ }^{2}$ Calli is the Venetian plural word for narrow streets - not to be confused with campi (pl.) or campo - used to describe the squares which break out from the calli, usually around churches.
} 
the origins of the word "rhythm"; from the Greek rhythmós related to rheîn meaning "to flow". Knowing this, it's satisfying to consider that Goethe's statement is in fact more loaded with meaning than it first appears. We are also reminded that architecture and music are not made of the same stuff, but compatibilities do exist and can be exploited in the composition and experience of each practice. Many composers seek out such compatibilities in numeric form, often in the translation of architectural, dimensional attributes into musical properties of pitch and time. In the writing of "Symphony no.3", 21 ${ }^{\text {st }}$ century composer, Sir Peter Maxwell Davies admits to "borrowing from renaissance architectural practice regarding 'vanishing points' in the planning of perspective... [to determine] harmonic design, over the largest of time-spans down to the smallest filigree detail". He also describes the intended experiential implications of this compositional device: "the ear traces an experience analogous to the eye's perception in a Brunelleschi church nave" (Davies, 2012). Similar to Dufay, Davies' application of Brunelleschi's geometric logic in the composition of Symphony no. 3 is purely analogical: However, Maxwell-Davies articulates an interesting distinction between ideas of form and ideas of architecture, with regard to the organisation of parts of a musical composition, according to their relative time-scales. Davies considers "form" as relating to the durations and ordering of sections of a piece, whereas the "architecture" provides the space in which these sections operate and interact: In this sense, the "architecture" of the music evokes a spatial dimension, which may be explained as providing a sense of "foreground, middleground and background"- whilst the "form"takes care of structuring the temporal relationships within the music. (Davies, 2013).

The metaphorical translations from the static, spatial attributes of Brunelleschi's great cupola to the spatially expressive, but invisible music of Davies" "Symphony no.3" are certainly deliberate and robust, however it seems there may be further opportunities to interrogate the potential for physical, acoustic resonances between the two pieces; with a performance of Davies' symphony within the space that it is designed to evoke. Davies is perhaps unknowingly choreographing a spatial overlay - where the spatial illusion of a place is conjured in parallel to the immediate experience of hearing the acoustic behaviour of the space in which the piece is being performed.

Many of my solo recorded pieces ${ }^{3}$ have been constructed from field recordings of harmonically or rhythmically rich sounds - such as boat engines, car brakes, singing vent shafts and even Geese grazing. Some of these sounds have a distinct connection to a specific place. For instance the low, but penetrating hum emitted by the chain Ferry which patrols the waterfront in Dartmouth, is used as a basis for the harmonies that develop throughout Dorsal Falls (V1). Even though these recorded sounds have been modified, the sound of the ferry engine may still be recognisable to anyone with a discerning ear (who has also spent some time in Dartmouth). During the process of re-interpreting these pieces for live performance, it's sometimes difficult to ignore the memory that's evoked of the places from which these sounds originate. This may be confusing when combined with the dynamics that occur from sometimes utilising the acoustics of a performance space which is different to that of the origin of the sounds. Though, as (perhaps accidently) illustrated in the work of sir Peter Maxwell Davies - folds upon folds of opportunities for exploration exist within the spaces between the place that is heard and the place that is remembered.

“Venice Spectrograph: 004”

\footnotetext{
3 | released two albums under the name EKM in 2015 - Pt.1 and 6:2
} 
The architectural proposals for an ongoing research project: The Augmented Instrumentalist (tAI), exploit the potential in this kind of superimposition - using it as a driver for the design of acoustically intelligent surfaces. This project projects forward to a time when the Venetian MOSE ${ }^{4}$ dam is complete and the phenomenon of the acqua alta is a fading memory, maintained only through anecdotal and photographic records. The architectural component of tAI proposes to replicate and thus preserve the immersive acoustic qualities of Venice in flood, by providing spaces which can mechanically replicate the behaviour of reflected sound in the city during the tidal phenomenon. Instrumental pavilions are located along the perimeter of the Venetian Lagoon and have both a visual and acoustic connection with the city. The pavilions simultaneously act as instruments, that physically simulate aspects of the current Venetian acoustic profile - and as auditoria, in which the sound producing elements of the Venetian soundscape (such as the church bells and fog horns of large cruise ships) are heard as they were at a time before the operation of the MOSE dam. Aside from their nostalgic function, to provide a historically-accurate acoustic backdrop, the pavilions - distributed along the coastline - have a distinctly musical function and are organised in a way which mimic aspects of the city's architectural spaces that influenced the development of Venetian polychoral music - a style which was highly influential in the evolution of Baroque music (Buelow, 2004: 17). A subset of that style, named cori spezzati (meaning separated choirs) reportedly arose from the unusual placement of double choir lofts which flank the altar of the Basilica San Marco in Venice. The separated choir lofts in this example presented composers with the opportunity to write pieces for two or more spatially fragmented choral groups. The 16th century composer Adrian Willaert was supposedly the first composer working in Venice to document the influence of this type of layout on the composition and choreography of his choral pieces. (Buelow, 2004: 17). Willaert's music considers buildings to have active acoustic operations beyond being mere receivers. This sentiment is recognised in the organisation of the surfaces and chambers which define the edges of tAl's instrumental pavilions. These spaces are designed to at once preserve a well-observed past condition and embrace uncertainty in providing the potential for interactive and improvised performances during future use.

“Instrumental Pavilion: Lido”

\section{Consequential resonance:}

The acoustic response of a room has the capacity to transform a musical experience, as identified by pioneer of architectural acoustics, Wallace Clement Sabine: "In any ordinarily bare and uncarpeted room, one may sing in succession a series of notes and then hear for some time afterward their full chordal effect" (Sabine, 1908: P845) - Sabine proposes that the acoustic characteristics of vernacular architecture across the globe has directly influenced the way in which musical styles have developed within respective cultures; specifically with regard to reverberance and the development of tonal music: "The reverberation which accompanied the lofty and magnificent architecture [in Europe] increased until even the spoken service became intoned in the Gregorian chant" (W.C. Sabine, 1908: 847).

“Doppler Swing”

\footnotetext{
${ }^{4}$ At the time of the Augmented Instrumentalist's inception - the MOSE (Modulo Sperimentale Elettromeccanico) dam project proposed to protect Venice and the coastline of the Venetian lagoon from tidal flooding, with the construction of 3 large, retractable dams at the lagoon inlets: Lido, Malamocco and Chioggia.
} 
In psychoacoustics, reverberation is the perception of the persistence of a sound beyond the point when the original emission finishes. Spaces bound by acoustically reflective surfaces tend to facilitate longer reverberation times as the reflected sound takes longer to decay. An echo is mechanically similar to reverberation, except the reflection of an echo is heard as a sound distinct from the original emission. The difference in the perception of these two phenomena lies in the time it takes for the reflection to reach the listener, and this time is directly attributed to room geometry. The threshold at which the perception of an acoustic reflection shifts between echo and reverberation is around 0.1 seconds, as this is the time at which sonic events cease to persist in human memory. We know that sound travels at approximately $343 \mathrm{~m} / \mathrm{s}$ in air, so we can define a distance of around 17 metres as the dimensional representation of that threshold.

My site-specific composition, Dorsal Falls V2, acknowledges the presence of reverberation (in the Church of St Leonard, Shoreditch) as a compositional device - to construct a series of unstable harmonies - the assembly period of which varies according to spatial details such as the position of the performer, the listener and the overall dimensions and geometry of the space. The composition process for Dorsal Falls V2 begins with 3D digital acoustic simulation models to visualise the path of the reflected component of five tones, each emitted from different parts of the building and over a time period determined by the reverberation time ${ }^{5}$ of the space (around 3 seconds). Once the corresponding notes of the chord are notated and assigned to their locations within the space, the drawing becomes a model for predicting both where and when the emitted tones will merge and be perceived as chordal by the listener. The resultant spatial score assists in improving an understanding of both the temporal and spatial implications of sound sources placed within a reverberant volume as well as providing instruction for the execution of the piece. However, its limitations must also be noted: The behaviour of polyphonic music in reverberant spaces is wonderfully complex and unpredictable, and this is celebrated in the iterative development of Dorsal Falls V2, through multiple, repeat performances. With each rendition, the piece will gradually become attuned to the space - supporting modifications to aspects such as the position and movements of the performers, durations of notes and maybe even the construction of some of the harmonies all together. These are changes that can't be predicted through a simplified, digital simulation model, or by rehearsing ideas through static and silent drawings. This kind of response must be discovered directly in the immediacy of the space, by playing the building and measuring its response in a more empirical manner - often resulting in the discovery and celebration of new subtleties and therefore adding richness to the overall piece.

“Animating reflectance: Sequence 010"

In the example of Dorsal Falls V2, the composition is simultaneously intended as an act of performance and an interactive mechanism for understanding the complex acoustic architecture of the space. Even though the piece is intended to be site-specific, it could still conceivably be adapted for performance in any reverberant space though this raises the question of - at which point does the acoustic behaviour of the space begin to transform the music into a new piece work? For instance, if Dorsal Falls V2 is played in the New Glyndebourne Opera house, the chordal blend may only last as long as 1.3 seconds as a result of the building's relatively short reverberation time. Even though the internal layout of the opera house is very different to that of the church, the perceived effect of the reverberation may be quite similar, as the difference in reverberation time between

\footnotetext{
${ }^{5}$ Reverberation time is the time it takes for a sound to decay by 60 decibels. This value changes according to dimensional and material characteristics and the frequency of the emitted tone. Sabine was the first physicist to develop a method for calculating reverberation time, in the 1890's using empirical methods for determining relationships between the volume of a room, its surface area and acoustic absorption (Sabine, 1908: 846)
} 
the two is relatively small. In contrast; the length of the chordal blends if performed in St Paul's Cathedral may last as long as 13 seconds (B.Shield \& T.Cox, 2000). Such a huge variation in decay-time is likely to drastically affect the character of the piece, beyond the abilities of the performers to adjust to the space. Granted, it's not unusual for the same piece of music to be performed in multiple locations with large variations in acoustic response, but it is seemingly rare that a composer or performer would admit to their work being susceptible to complete transformation, owing to this sort of variation.

On a much smaller scale, micro adjustments are often made in the times at which different members of an orchestra play their notes, depending on their individual proximity to the audience. This is to ensure that sounds which are intended to be heard in unison, but are emitted at a distance from each other, end up reaching the listener at the same time. These adjustments operate in denominations of time which are too small to count, but can nevertheless still be discerned. Such subtle adjustments are usually not explicitly notated in the score, but instead 'read' in the geometry of the performance space by the conductor and performers. ${ }^{6}$ It seems that this kind of practical acoustic knowledge amongst musical performers is often tacitly understood and developed through years of rehearsal and experience, as opposed to being formally learned as a science or worked out in advance using computational tools. Conversely, and from the position of the designer of the space, it would seem that many architects and engineers appear to privilege the explicit science of acoustics over empirical evidence inferred from the recollection of acoustic experiences. Architect and historian Michael Forsyth argues the case that the practice of acoustic design is "a matter of musical judgement rather than simply a scientific process" (Forsyth, 1985: 16) and until Sabine founded the field of modern architectural acoustics with a series of influential scientific publications in the 1890's, the design of concert hall and theatre auditoria was arguably largely an exercise of trial and error, through what Forsyth describes as "a combination of intuition, experience and luck" (Forsyth, 1985: 13). Since the birth of modern acoustic sciences, we have undoubtedly gained the ability to more precisely predict how sound will behave in spaces, and the tools for visualising otherwise invisible, sonic phenomena - however, we may have in-turn lost the desire to trust our discovery of these behaviours through experience and thus the ability to immediately observe and respond to unexpected and often subtle events. This immediacy is what fuels the forging of rich, creative relationships between sound and space. Our so-called buildings for music have been reduced to passive containers and are no longer charged with the acoustic intelligence that becomes apparent when treating them as compositional devices - as we would musical instruments. Deleuze speaks of this kind of musical interactivity "the sources of sounds... are not content only to send sounds out: each one perceives its own, and perceives the others while perceiving its own" (Deleuze, 1993: 91) - this sentiment could be extended to encompass all active operators within a performance (not just the ones which generate the sound). This reciprocality is available to be creatively discovered - both in the way that we consult sound as architects - and space as musicians.

“Sketch for a Spatialised Score"

One of the most intriguing, early examples of acoustically-intuitive architectural design is Vitruvius' proposal for the use of "Echea", or acoustic vases, in theatres. In chapter five, book five of the "Ten Books on Architecture" Vitruvius describes a precise system for organising the geometry and spatial placement of these vases, with the conviction that the air within these vases, in their respective locations around the auditoria, will become excited (resonate) when they are "touched"by the wide spectrum of sonic frequencies emitted by performers on the

\footnotetext{
6 I became aware of this during an informal discussion with K. Yusuf - composer, performer and co-founder of the London Graduate Orchestra. Also a tutor at the London school of the Arts.
} 
stage (Vitruvius, 1486: 143-150). There are apparently no definite, remaining examples of these acoustic devices (Godman, 2016), so it is difficult to understand exactly how effective they were in action, but Vitruvius nevertheless made a convincing case, for appreciating the potential for a building to behave as an instrument in its own right. More recently, Alvin Lucier's "I am sitting in a room" (1969) exists as a successful, example of a model for musical performance where the room is explicitly appropriated as an instrument. Lucier repeatedly rerecords a tape of his own voice as it plays back in a room. With each iteration of the recording, the resonant profile of the room enhances certain frequencies present the recording and gradually the recital of the text is transformed into pure tones, which form the acoustic signature of the room's resonant capabilities. The result of this performance will undoubtedly vary between rooms as this piece is inherently site- specific. It's interesting to consider the potential for reverse engineering such recordings - perhaps with the intention of reconstructing the architecture of the room in which it was recorded - at least in terms of its dimensional, geometric and material attributes. Lucier-like sound profiles could eventually act as (sonic) design briefs for architects of 'spaces for music' - directed by the resonant requirements of the musicians who wish to commission them.

“Interval Scatter"

\section{Sculpting Resonance}

Acousticians at Arup have developed an analytical, acoustic design tool called the "SoundLab"- An immersive test-space which allows its occupants "to listen to buildings before they are built... The aural equivalent of a visual render in architecture... which allows subjective design decisions to be made" (Patel, 2015). In this example, an anechoic sound is digitally manipulated and then spatially and temporally re-mapped to a series of speakers which line the interior of a spherical space, at the centre of which, the listener is located. This tool attempts to combine the computational precision of multi-dimensional digital simulation with the richness of real-time perceptual analysis, to allow both architects and musicians to practice an acoustic reality in advance of realising their vision.

Since Sabine's discoveries, methods for predicting the behaviour of sound in space have become increasingly sophisticated. Digital simulation tools allow us to visualise and recalibrate our understanding of acoustic behaviours, as non-linear events and at timescales small enough to be imperceptible. This is unquestionably useful in allowing us to interrogate, rehearse and refine a condition before it gets committed to the material, linear real-time world, however these elastic virtual realities are still unable to fully replicate the complexities of the emergent properties in real-life experiences. This is not surprising when we consider the potential variety of listener experiences with regard to psychoacoustics, combined with multiple variations in material environments - all of which which have a collective effect on the way in which sound travels through space in the time between leaving an emitter and arriving at the listener's ear. The certainty and predictability we are promised in these digital rehearsals, is sometimes short-sighted and often forgets the creative potential in understanding the physicality and potential for improvisation within this inherently unpredictable condition.

During the early stages of tAI, countless digital simulations were produced to determine the behaviour of reflected sound in specific areas of Venice, in order to generate 3D sound profiles, around which surfaces were wrapped and architectural ideas were developed. However, it's easy to forget that digital models are often reductive - in terms of the physical behaviours that they describe - and it quickly becomes necessary to construct and consult physical prototypes in order to gain complex, immediate, 1:1 experiential feedback. The methodology of combining feedback from physical tests with that gained from digital simulation makes for a 
comprehensive - albeit not complete - idea of how the invisible material of sound will behave in a series of spaces that don't fully exist, other than theoretically or partially in drawings, scores, mockups, calculations and textual descriptions.

“The Waffle”

The Waffle is a prototype, fabricated at 1:1 to test a portion of the modular, acoustic wall system that is proposed in drawings and digital models for tAl. The geometry of the piece was initially designed using feedback from digital simulations. The sizes and shapes of the surfaces and voids, correspond to the wavelengths of tones that commonly occur in the Venetian soundscape (determined from spectral analysis of sound recordings, captured during my visits to Venice).

“Spectral and Reflectance Profile Modelling"

The parts for the main Steel structure are digitally modelled and laser cut (to ensure accuracy) then assembled carefully, by hand. Since assembly, the piece has displayed some curious characteristics that (perhaps unsurprisingly) hadn't been anticipated in the virtual version. For instance; the high-frequency squeaking and rattling that occurs in the connections between panels as a result of the tolerance around the slots that are required in order to slide two pieces together. Also, considerable deformations in the overall shape of the piece due to self-weight. The tolerance in the slots between the sheets of the main structure, allows the piece to move and change its shape depending on where and how it is being supported. Of course, these formal discrepancies have an affect on the acoustic performance of the piece and this raises the question of whether the resultant pliability of the piece should be fixed, or taken advantage of - especially considering the depths of the voids had originally been calculated to respond to specific frequencies. In truth, the curiosity to exploit this unexpected flexibility and explore the piece as a new, active and adaptable tool for responding to variable sonic stimuli, overwhelms the desire to loyally complete the construction of the prototype as the digital model had promised. Afterall, what is the point in conducting an experiment if you know exactly what is going to happen? This project not only celebrates adaptability and interactivity in the context of an architectural proposal, but also in the dynamic methodology of research through design, and designing through making. The spatial and sonic uncertainties observed when rehearsing with physical prototypes, give rise to compositional opportunities in architecture and music, respectively.

“The Waffle: Resonance Analysis"

\section{Ideological resonance:}

Composers commit musical ideas to a score, in an attempt to record and preserve creative intent across performances. The score for Dorsal Falls V2 is unlike a conventional musical score. These drawings not only communicate the pitch, rhythm, dynamics and order of a series of sounds - they simultaneously provide instructions for their spatial coming-into-existence. The resulting documents occupy a realm somewhere between traditional Western music notation, architectural drawing and choreographic drawing. Similarly, contemporary composers and choreographers such as John Cage and Rudolf Von Laban have invented their own graphic methods to augment or replace traditional ways of notating the intangible. However, all of these approaches are still limited in their capacity to communicate the exact subtleties of expression intended by the composer. As architect Stan Allen identifies: "notations are necessarily reductive and abstract... [notation is] better able to anticipate the complexity and unpredictability of the real"(Allen, 2000: 42) With this in mind, 
drawings produced to facilitate any musical composition and performance are means to an end and not ends in themselves. In the case of Dorsal Falls these drawings provide a set of visual cues for understanding and predicting aspects of an otherwise indeterminate future condition.

"Site Sketch"

Even with the score and composer present, no two performances are performed or experienced the same. There exist perceptive variations, in how expressive aspects of notations are interpreted; in how the performer physically interacts with an instrument; in the expectations, tastes and acoustic memories of the listener - and also environmental variations, in the speed of sound due to air temperature and humidity; in the acoustic absorbency of the room due to the number of occupants, their distribution and the qualities of their clothing; even in the geometry of the anatomy of each listener's auditory canal - just to name a few. It's impossible to control the quantities, configurations and intensities of these unpredictabilities, but it is possible to expose and to take advantage of them.

Improvised music embraces uncertainty - it understands the fact that no two performances of the same piece are ever performed or experienced in the same way. Improvised music is usually founded upon base sets of rhythmic, harmonic and melodic structures, set out by the composer/s. From these points of departure, musicians are free (to varying degrees) to interpret and musically explore the themes laid out in these structures. However, the territories of these explorations rarely extend beyond the instruments, to explicitly engage with and allow the acoustic responses of the space to influence the direction of the music. If we consider the instrument and the space as part of an acoustic continuum - as opposed to two distinct entities the idea that the space can be an active agent in the improvisation of music, becomes easier to comprehend.

The documents which set-out the instructions for the performance of Dorsal Falls V2, contain explicit information about the harmonic, melodic and rhythmic components of the music - and also suggestions for positions of the performers and audience, within the performance space. This 'spatialised' score is not an attempt to guarantee a set of uniform recitals, however it is intended to act as a guide for discovering and understanding the acoustic peculiarities of a performance space. In this sense, the piece is 'site-specific' as it iteratively evolves, over time, with each performance and in response to the acoustic signature of the space. The score is not the music and "no piece is complete until it has been performed" (C.Wolff, 2012: 95).

\section{"Site Score"}

The drawing "Site Score" describes how notational drawings for the performance of Dorsal Falls V2 will be projected directly onto the site, as devices for suggesting - but not imposing - a positional hierarchy with regard to locating performers and listeners. In this example, the anamorphicized projection of the notation offers a fragmented view of the score from any viewing position other than the suggested location of the performer (set out by the drawing). Both the music and its visual instruction start to assemble at choreographed points within the space. This drawing exists as an experiment for encouraging the discovery of the performance space and the discovery of the music, simultaneously. The score exists as a spatial and musical guidance for performers, in the understanding that each recital of the piece will be different, but based upon the same base rules. 
The "Folded Score" is an experimental, double-sided drawing which intends to enable the simultaneous discoveries of pre-written musical motifs and architectural space, through interaction which mimics gameplay. The hope is that the performers will gradually develop an understanding of both the possible musical developments of the piece and the acoustic response of the different parts of the space in which they are playing, by continually folding and unfolding the drawn score, to reveal the next set of instructions. The combinations of possible outcomes are unavoidably limited according to the number of folds and the geometry of the folds, but the possible sequence of outcomes are expected to result in a series of performances (of the same piece) - which will vary according to these 'on the spot' decisions required of the performers in order to navigate both the piece and the space.

\section{Concluding resonance:}

When we consider performance spaces to be as acoustically active as the instruments which they contain, ideas surrounding the composition of space and music start to become more pliable. The space and the listener are as completely implicated in a musical performance as the instruments which initially produce the sound. By first acknowledging and then interrogating the interdependence inherent in the relationships between these agents, we can begin to use music as a mediator - a medium for negotiating boundaries between sound, space and time - and for boundaries within the music itself. My architectural research project, tAl and musical research project, Dorsal Falls - are developed in tandem, as a means of developing a multidisciplinary method of practice which sits within the seams between architecture and music. My research proceeds through the development of iterative design projects, performances and prototypes. The design projects and performances offer opportunities to test and refine physical and conceptual aspects of work. In order to develop these ideas in both a rigorous and experimental way, it's necessary to combine methods of digital modeling and simulation with physical prototyping and feedback from real-time performances. Interaction, improvisation and the exploitation of uncertainty form key themes in the work. These themes acknowledge the ephemeral nature of the experience and navigation of both architectural space and performed music. Importantly, these themes also celebrate opportunities for developing a mutually productive dialogue between these two, otherwise opposing practices.

“The Construction of Unstable Harmonies"

\section{References:}

Rasmussen, S.E. (1962) Experiencing Architecture. Cambridge, MA, United States: M.I.T. Press. Print.

Goethe, J.W.V, Fuller. M, and Eckermann. J.P, (1839) Conversations With Goethe In The Last Years Of His Life. Boston: Hilliard, Gray, and Company. Print.

Warren, C.W. (1973) Brunelleschi's Dome and Dufay's Motet. The Musical Quarterly, LIX(1), pp. 92-105. doi: 10.1093/mq/lix.1.92. Print.

Evans, R. (1995) The Projective Cast: Architecture and its Three Geometries. Cambridge, MA: MIT Press. Print.

Maxwell Davies. P. (07/2012) Symphony No. 3 / Cross Lane Fair, BBC Philharmonic. Catalogue No: 8.572350. Naxos Physical Release. Liner notes. 
Maxwell Davies. P. (13th June 2013) Changing the face of 'new' music. University of London. Lecture.

Buelow, G.J (2004) A History of Baroque Music. Bloomington \& Indianapolis: Indiana University Press. Print.

Sabine, W.C.W. (1908). Melody And The Origin Of The Musical Scale. Cambridge: [publisher not identified]. Print.

http://www.acoustics.salford.ac.uk/acoustics_info/concert_hall_acoustics/?content=reverb - (accessed June 2016). Web.

Forsyth, M. (1985). Buildings for Music - Architect the Musician \& Listener From 17 Century to Present. Cambridge, MA: MIT Press. Print.

Deleuze, G. and Conley, T. (2006) The fold: Leibniz and the baroque. New York: Continuum International Publishing Group. Print

Vitruvius, P - translated by Morgan, H.M (1914 - originally 1486) The Ten Books on Architecture. Cambridge: Harvard University Press. Print.

Godman, R. (2016) The Enigma of Vitruvian Resonating Vases and the Relevance of the Concept for Today. Hertfordshire: The Music Centre, Faculty for the Creative and Cultural Industries, University of Hertfordshire. Print.

Arup. (2015) A global firm of consulting engineers, designers, planners and project managers. Available at: http://www.arup.com/services/acoustic_consulting/soundlab_overview - (Accessed: 3 July 2016). Web.

Allen. S (2000). Practice: Architecture, Technique and Representation Psychology Press. Print

Hicks, M. and Asplund, C. (2012) Christian Wolff. United States: University of Illinois Press. Print

\section{List of Illustrations}

“Reflectance Profile 001: Chioggia” Emma-Kate Matthews, 2012

"Annotated Spectrograph: 004" Emma-Kate Matthews, 2012

“Instrumental Pavilion: Lido” Emma-Kate Matthews, 2013

“Doppler Swing” Emma-Kate Matthews, 2016

“Animating Reflectance: Sequence 010" Emma-Kate Matthews, 2015

“Sketch for a Spatialised Score" Emma-Kate Matthews, 2016

“Interval Scatter" Emma-Kate Matthews, 2016

“The Waffle" Emma-Kate Matthews, 2012

"Spectral and Reflectance Profile Modelling" Emma-Kate Matthews, 2013

“The Waffle: Resonance Analysis" Emma-Kate Matthews, 2012

"Site Sketch" Emma-Kate Matthews, 2015

“Site Score" Emma-Kate Matthews, 2016

“Folded Score" Emma-Kate Matthews, 2016

“The Construction of Unstable Harmonies" Emma-Kate Matthews, 2017 


\section{Contributor details:}

Emma-Kate is an architect, artist and musician. She founded EKM Works in 2012; a multidisciplinary, solo practice which has exhibited work in the Royal Academy and has had work published by Wiley and Thames \& Hudson. She currently teaches an MArch unit at the Bartlett school of architecture - focussed on the practice of designing through making. Emma-Kate is due to start a PhD at the Bartlett School of Architecture in the summer of 2017 which will explore themes of site-specific musical composition, performance and spatial choreography.

http://ekm.works

http://ekmusics.bandcamp.com

hello@ekmworks.com 